Historic, Archive Document

Do not assume content reflects current scientific knowledge, policies, or practices. 



\section{THE HOME OF}

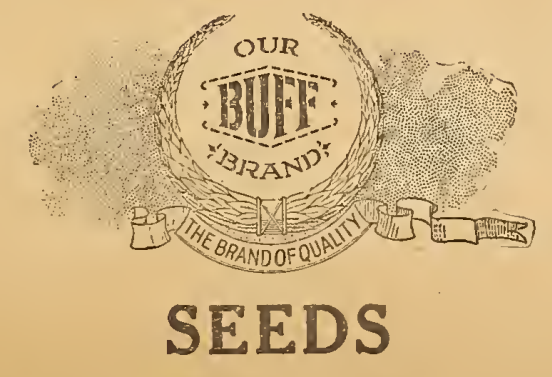

62.37

JOHN J. BUFFINGTON \& CO. SEED MERCHANTS

BALTIMORE - - - MARYIAAND 



\section{JOHN J. BUFFINGTON \& CO. WHOLESALE SEED MERCHANTS

\author{
$108-118$ LIGHT STREET
} \\ BALTIMORE, MD., FEBRUARY 4, 1927.}

Markets continue frm, with a few advances in price. There has been quite an improvement in both inquiries and orders this week-sales the largest of any week this season.

With continued nice weather we expect an early active demand. We can supply you with both the DOMESTIC and FRENCH grown Red Clover to advantage. The FRENCH SEED we consider siplendidly adapted to your conditions. Will appreciate your inquiries and send samples when desired PHONE, WIRE or WRITE US, WE CAN PLEASE YOU-QUALITY, PRICE, SERVICE.

We do not handle Italian or South European grown Red Clover or Alfalfa.

\begin{tabular}{|c|c|}
\hline \multicolumn{2}{|l|}{$\begin{array}{l}\text { RED CLOVER } \\
\text { ADAPTED-FRENCH GROWN }\end{array}$} \\
\hline & Lb. \\
\hline Prime................. & .27 \\
\hline Strictly Prime.............................97.00\% & .291 \\
\hline 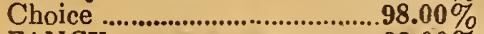 & .30 \\
\hline FANCY & .31 \\
\hline BUFF ..........................................99.50\% & .32 \\
\hline
\end{tabular}

FANCY..............

RED CLOVER

UNITED STATES GROWN

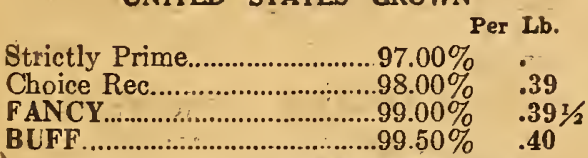

SAPLING OR MAMMOTH

UNITED STATES GROWN Per Lb.

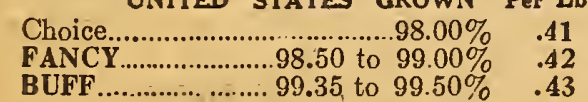

ALSIKE CLOVER

$\begin{array}{ll}\text { Choice........................95.00 to } 96.00 \% & .35 \\ \text { FANCY................... } 96.50 \text { to } 97.00 \% & .36\end{array}$

$\begin{array}{lll}\text { FANCY.........................9. } 98.50 \text { to } 97.00 \% & .36 \\ \text { B UFF.................... } 98.00 \text { to } 98.50 \% & .37\end{array}$

ALFALFA

UNITED STATES GROWN Per Lb.

Choice …………………..... $98.50 \%$

BUFF........................................ 99.50\% .189

KANSAS ALFALFA

BUFF .................................. 99.50\% .20

NORTHWESTERN ALFALFA

BUFF $99.50 \% \frac{\text { Par. } .26}{.201 / 2}$

CANADIAN VARIEGATED

BUFF............... Certified ....................... Per Lb. GRIMM'S ALFALFA CLOVER

BUFF............ Certified.......... $99.50 \%$

CRIMSON CLOVER

FANCY Per Lb.

WHITE CLOYER

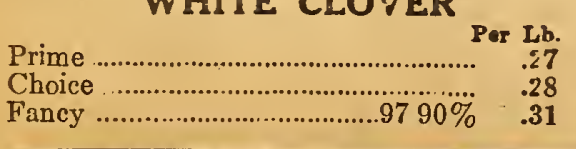

HUTOEERII

Insures Puicker Catch-Bigger Crops

\section{CLOVERS}

Wholesale Retail

\begin{tabular}{rrrr}
1 Bu. Onit & $\$ 1.50$ & & $\$ 2.25$ \\
$1 / 3$. Bu. Dnit & .40 & 1.00 \\
$1 / 4$ Bu. Unit & .24 & .60 \\
\hline
\end{tabular}

SOY BEANS - COW PEAS - VETCHES

$\begin{array}{lcc} & \text { Wholesale } & \text { Retail } \\ \text { B Bu. Unit } & \$ 1.50 & \$ 2.25 \\ \text { 1 Bu. Unit } & .40 & .60 \\ \text { \% Bu. Unit } & .24 & .85\end{array}$

State desired variety.

Posters and Leaflets on request.
SWEET CLOVER

$\begin{array}{cc}\text { SCARIFIED } & \text { Per Lb } \\ \text { WHITE BLOSSOM Choice.............. } & .12\end{array}$

WHITE BLOSSOM Fancy. $.98 .50 \% \quad .131$

GRUNDY COUNTY........................ $\quad .151 / 2$

YELLOW BLOSSOM Buff.................

TIMOTHY

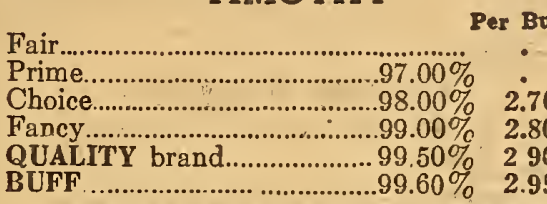

\section{KENTUCKY BLUE GRASS}

Choice Kentucky .................8 $8000 \%{ }^{\text {Per }}{ }^{\mathbf{L} b .}$

Fancy Kentucky .................85.00\% .24

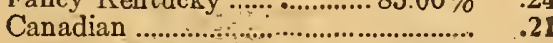

\section{ORCHARD GRASS}

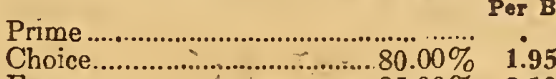

Fancy............................................85.00\% 2.10

BUFF ........... $90.00 \% \quad 2.20$

\section{RED TOP/HERD GRASS}

Choice Fancy.........................90.00\% Per Lb. BUFF Fancy......................... 92.00\% . .2

Choice Unhulled

Fanev Unhulled.

RYE GRASS

Perennial...............................98.50\% Per Lb.

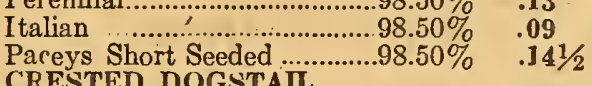

CRESTED DOGSTAIL

HARD FESCUE.

SHEEP FESCUE
RED FESCUE....

\section{PERMANENT PASTURE}

For Upland..................................... .12 Per $\mathrm{Lb}$ to GERMAN MILLET

Choice

Choice.

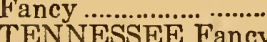

WHITE WON DER (large growth) HUNGARIAN

\section{SORGHUM OR CANE}

Fancy Amber Per Bu

Fancy Orange........................................... 195

ALASKA PEAS (Sealed).... 6.60

\section{SEED POTATOES} (MAINE GROWN)

Irish Cobblers................11 Peck Sack 6.50 Early Rose.....................11 Peck Sack 650 Red Bliss.......................11 Peck Sack 650 Beauty of Hebron...........11 Peck Sack Crown Jewell

Green Mountain..............11 Peck Sack 6.50

BUFF BRAND POULTRY FEEDS SCRATCHING SIZE

10 sack lots........................per 100 lbs. 235 Per 100 lb. sack....................per 100 lbs. 240 CHICK SIZE

10 sack lots.......................per 100 lbs. 250 Per $100 \mathrm{lb}$. sack.................. per 100 lbs. 255

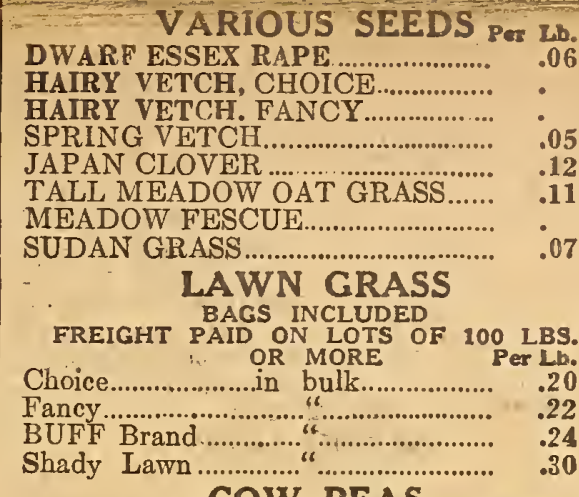

\section{COW PEAS}

RECLEANED, SACKS FREE $\quad$ Por Bi
Mixed..................................................
2.10

Whippoorwill Mixed..............................

Gray Whippoorwill

Blacks...

New Era......................................... 265

Groit.....

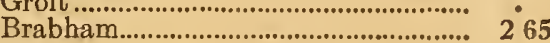

Clay.

Gray Crowder

Black Eye, Virginia.................
COW PEAS and SOJA

Mixed......

$$
\text { SOJA BEANS }
$$

RECLEANED, SACKS FREE Par Ba
Mammoth Yellow.

Manchu.

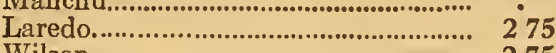

Wilson........................................................... 2.75

2.75

Hollybrook.

Tar Heel.

$$
\underset{\text { AMERICAN GROWN PAS }}{\text { CANA. }}
$$

Fancy

\section{SEED GRAIN}

BAGS EXTRA Per Bo

Rosen Seed Rye

Abruzzi Seed Rye

Buckwheat. Japanese...

FULTZ WHEAT

Leafs Prolific Wheat.

China Wheat

BARLEY

White Spring Oats

Winter Seed Oats (Virgini............... $\quad .75$

urt Oats

NSILAGE CORN (Southern White)

\section{MISCELLANEOUS FEEDS}

DRY MASE ................... per 100 lbs. 2.50

Reef Scraps................50\% per 100 lbs. 375

Crushed Bone......................per 10 lbs. 3.00

Meat Meal...............50\% per 100 lbs. 3.75

Digester Tankage..60\% per 100 lbs. 3.75

Kaffir Corn....................................per lb. 100 lbs

Hemp ...............................................

Buckwheat.........................................per lb. :03

Vetch..................................... per lb. 100 lbs. 2.30

Canada Field Peas.....

Millet.......................................per lb.

Alfalfa Meal............................ 100 lbs. 2.20

CRUSHED OYSTER SHELI

Ton lots...

10 sack lots

10 sack lots..............

We ouote Firm Prices by wire, when desired, on seeds, car lots or less. 


\section{LAWN GRASS AND PASTURE MIXTURES}

\section{QUALITY IS OF GREATEST IMPORTANCE}

OUR LAWN AND PASTURE MIXTURES ARE COMPOSED OF SEEDS OF THE BEST QUALITY AND IN PROPER PROPORTIONS.

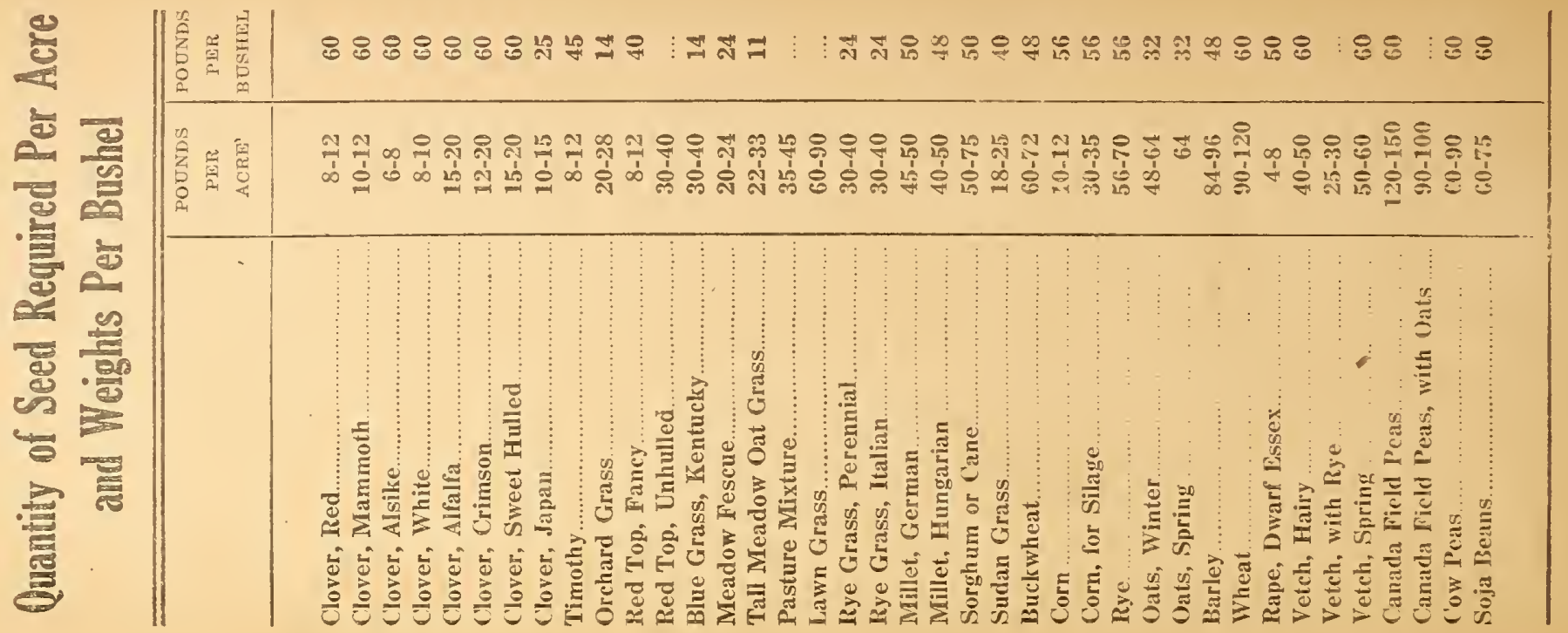

